\title{
The value of magnetic resonance imaging in evaluation of myocardial and liver iron overload in a thalassaemia endemic population: a report from Northeastern Thailand
}

\author{
Narumol Chaosuwannakit ${ }^{1}$, Pattarapong Makarawate ${ }^{2}$ \\ 'Department of Radiology, Faculty of Medicine, Khon Kaen University, Thailand \\ 2Department of Internal Medicine, Faculty of Medicine, Khon Kaen University, Thailand
}

\section{Abstract}

\begin{abstract}
Purpose: Patients with chronic haemolytic anaemia, such as in thalassaemia, require repeated blood transfusions, which leads to iron overload and cellular damage, especially in the heart and liver. Classically, serum ferritin and liver biopsy have been used to monitor patient response to chelation therapy. Magnetic resonance imaging (MRI) has proven to be effective in detecting and quantifying iron in the heart and liver. The aim of the paper is to evaluate the accuracy of the MRI T2* procedure in the assessment of liver iron concentration and myocardial iron overload. Material and methods: In 210 cases of monthly transfused patients, hepatic and myocardial iron overload was measured by multi-breath-hold MRI T2* and compared to serum ferritin (a traditional marker of iron overload).
\end{abstract}

Results: No significant correlation was observed between serum ferritin level and cardiac T2* MRI $(p=0.68$, $r=0.06)$. However, a significant correlation was observed between serum ferritin and liver iron concentration evaluated by MRI ( $p=0.04, r=0.68)$.

Conclusion: Routine evaluation of liver and heart iron content using MRI T2* is suggested to better evaluate the haemosiderosis status in thalassaemic patients.

Key words: MRI, iron overload, thalassemia, cardiac MRI, liver iron concentration, T2*.

\section{Introduction}

Iron is an essential element that forms a vital component of metabolic and biological processes, but when present in excess it can yield tissue damage due to oxidative stress. Patients with thalassaemia require regular blood transfusions to survive beyond the second decade of life [1-3]. Even though transfusions improve health and survival, the chronic administration of large amounts of blood, along with extra vascular haemolysis and an increase in the intestinal absorption of iron, inevitably leads, despite chelation therapy, to significant haemosiderosis of all organs [4]. Excess body iron may accumulate in the liver, spleen, heart, bone marrow, pituitary, pancreas, and the central nervous system, initiating damage to these organs [5]. Iron overload cardiomyopathy (IOC) results from the accumulation of iron in the myocardium, and it frequently ensues late and can be difficult to reverse once established, and it is a significant cause of death in patients in receipt of chronic blood transfusion therapy [6,7]. Inopportunely, significant cardiac iron burden can happen regardless of low liver iron concentration [8]. There is significant clinical importance to reliably risk stratify patients for the development of heart failure, so that chelation therapy can be increased and cardiac morbidity averted [9]. Indirect markers of current cardiac iron overloading, namely

Correspondence address:

Narumol Chaosuwannakit, Khon Kaen University, 499/132, Mittraparb road, Muang district, 40000, Khon Kaen, Thailand, phone: 66846464640,

e-mail:narumol_chao@yahoo.com

Authors' contribution:

A Study design · B Data collection · C Statistical analysis · D Data interpretation · E Manuscript preparation · F Literature search · G Funds collection 
single measurements of serum ferritin and biopsy-determined liver iron concentration, are inadequate for identifying myocardial iron deposition in chronically transfused patients $[1-3,10]$.

Ferritin is a metalloprotein that is found in cells. It stores and releases iron in a controlled manner. In normal individuals, a small amount appears in the circulation and in general reflects the total body iron. Normal values of serum ferritin for men and women are 12-300 ng/ml and $12-150 \mathrm{ng} / \mathrm{ml}$, respectively [1-3]. It is an acute phase reactant, and the serum levels may be disproportionately greater than the degree of iron loading in infections, inflammatory states, liver dysfunction, and malignancies [1-3]. However, magnetic resonance can measure the paramagnetic properties of tissue iron to provide direct, non-invasive iron assessments, and clinically this has been achieved by assessing the $\mathrm{T} 2$ and $\mathrm{T} 2{ }^{\star}$ relaxation parameters [11]. Magnetic resonance does not image the iron directly but instead images water protons as they diffuse near iron deposits in the tissue of interest [8]. The iron acts as small magnets, extinguishing the homogeneity of the magnetic field in iron-laden tissues. The moving water protons experience significantly dissimilar magnetic profiles and become desynchronised from one another. This causes the image to darken at a level proportional to the iron concentration [12]. This technique is unassuming, allowing fast and reproducible quantification of myocardial and hepatic iron, consequently allowing pre-symptomatic detection of myocardial siderosis [13]. The T2* technique has the potential to become a noninvasive standard technique in evaluating tissue iron [10-12]. In the present study, we also compared the MRI T2* results with serum ferritin levels of patients on regular blood transfusion.

\section{Material and methods}

Cardiac $\mathrm{T}^{*}$ and liver iron concentration (LIC) measurements were performed on 210 thalassaemia major patients (110 males and 100 females, age $11.1 \pm 9.8$ years) who received regular transfusion and iron chelation therapy with serum ferritin levels $>1000 \mathrm{ng} / \mathrm{ml}$. Patients with a contraindication to magnetic resonance imaging, including pacemakers, claustrophobia, and inability to comply with the instructions, were excluded from the study. Approval was obtained from the Ethics Committee of the Faculty of Medicine.

\section{Magnetic resonance imaging interpretation}

The images were acquired on a 1.5-T Avanto system (Siemens Medical Solutions, Erlangen, Germany) following a standard protocol system using a cardiac phased-array coil. Each patient was scanned using both the optimised bright-blood [14,15] and the original black-blood sequences [16]. In summary, each technique is a cardiac-gated, multi-echo, fast gradient sequence acquired within a single breath-hold. The black-blood technique employs an additional double inversion recovery prepulse to null the blood signal in the heart chamber. A single midventricular short-axis slice was acquired with a slice thickness of 6 and $10 \mathrm{~mm}$ for the black blood techniques. Imaging parameters were a TR of $19 \mathrm{~ms}$, eight echo times (2.616.74 ms with 2.02-ms steps), a matrix of $128 \times 256$, and a FOV of $40 \mathrm{~cm}$, which yielded a voxel size of $3.1 \times 1.6 \times 10 \mathrm{~mm}^{3}$, while the inversion time (TI) was set to suppress the blood signal. The acquired images were analysed locally and also transferred in DICOM format to be processed at the reference site. All T2* analyses were performed independently at the site using individual, custom software developed in MATLAB (The MathWork, Natick, MA) [14,15]. All image data were fit to the offset model at the reference site (REF-offset) and fit to the offset (LOC-offset) and mono-exponential (LOC-typical) models at the local site. ROIs had been defined, manually, from the whole interventricular septum (WS) at the reference site, as in the conventional method, and from the partial interventricular septum (PS) region using prior knowledge of a $\mathrm{T} 2{ }^{\star}$ map to avoid susceptibility artefacts and partial volume effect at the edge of the septum, as suggested in previous studies [14-16]. A radiologist with more than 10 years' experience in cardiac MR analysis independently performed the analyses. The $\mathrm{T} 2{ }^{*}$ results of all models in this study were reported by using both their mean and median values. The result from a typical LOC method reported by its median is, therefore, equivalent to the MPS-PW method. For the measurement of liver T2*, a single trans-axial slice through the centre of the liver was imaged at eight echo times (2.3-16 ms). The TR was set to $200 \mathrm{~ms}$ without cardiac gating. Signal intensity analysis was performed in the periphery of the liver away from the large central vessels.

The cut-off points in this MRI instrument were as follows for liver iron concentrations (LIC): normal $\leq 3 \mathrm{mg}$ Fe/g dw, mild 3-7 mg Fe/g dw, moderate 7-15 mg Fe/g dw, and severe $\geq 15 \mathrm{mg} \mathrm{Fe} / \mathrm{g} \mathrm{dw}$ (Figure 1).

Cardiac T2*: normal $>25 \mathrm{msec}$, marginal $20-25 \mathrm{msec}$, mild to moderate $10-20 \mathrm{msec}$, and severe $\leq 10 \mathrm{msec}$ (Figure 2).

\section{Serum ferritin levels}

Measurements were carried out using a microparticle enzyme immunoassay (MEIA; Abbot Diagnostics, IMX System, Ferritin, IL, USA).

\section{Statistical analysis}

Statistical analysis was performed with commercially available software (SPSS Inc., version 18.0, Chicago, IL, USA). A paired Student's $t$-test was selected to evaluate the difference between the two data. A $p$ value less than 0.05 was considered to be significant. 

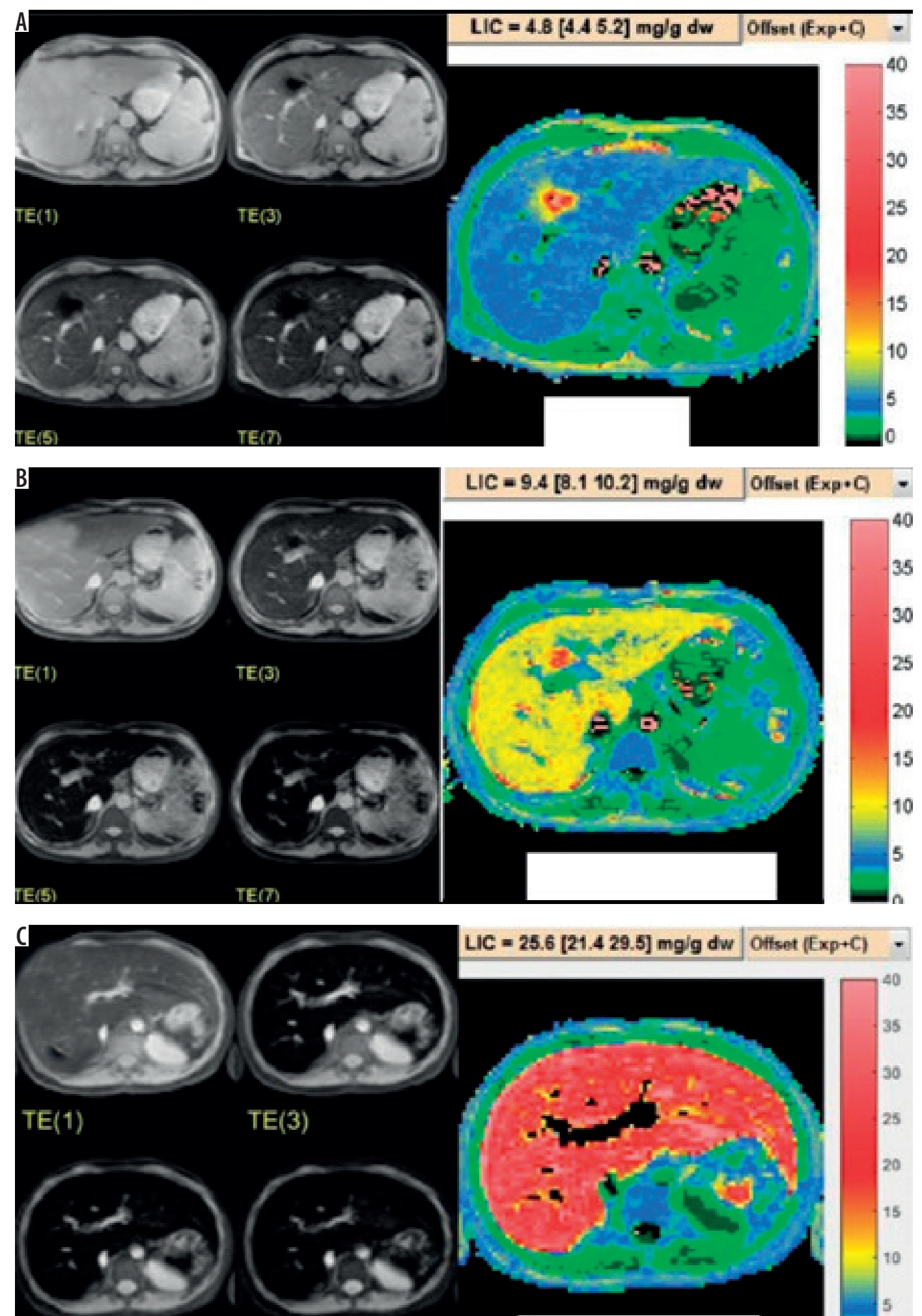

$\mathrm{TE}(3)$

\section{TE(5)}
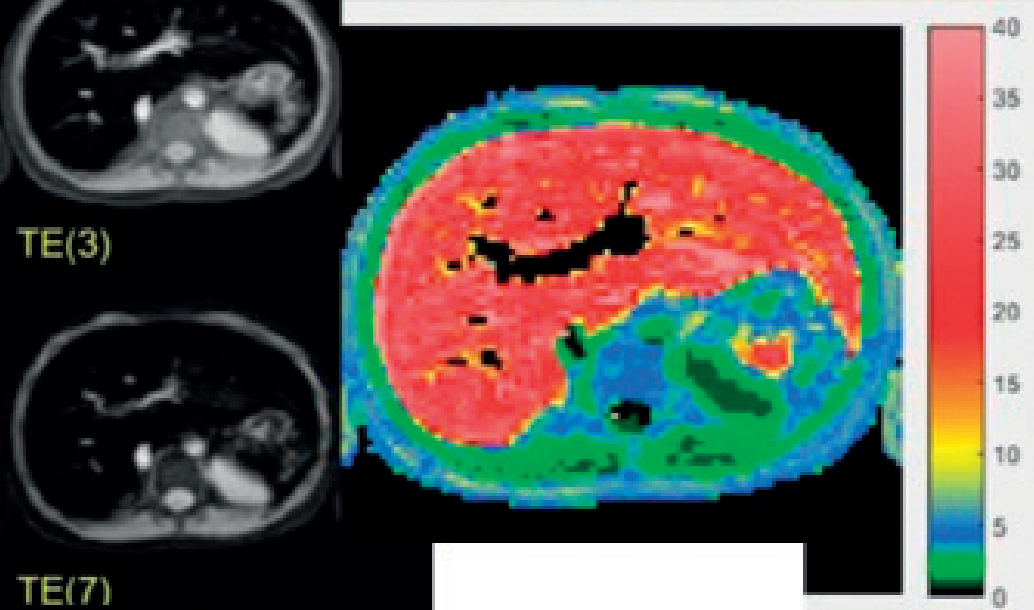

Figure 1. Example of liver iron concentration evaluated by MRI from mild (A), moderate (B), and severe $(C)$ liver iron overload thalassaemia patients

\section{Results}

\section{Patients}

Cardiac $\mathrm{T}^{*}$ and liver iron concentration measurements were performed on 210 thalassemia major patients (110 ma- les and 100 females, age $11.1 \pm 9.8$ years). Splenectomy had been performed in 12 of the patients. All patients had received iron chelation therapy from early childhood for a median duration of 18 months. 

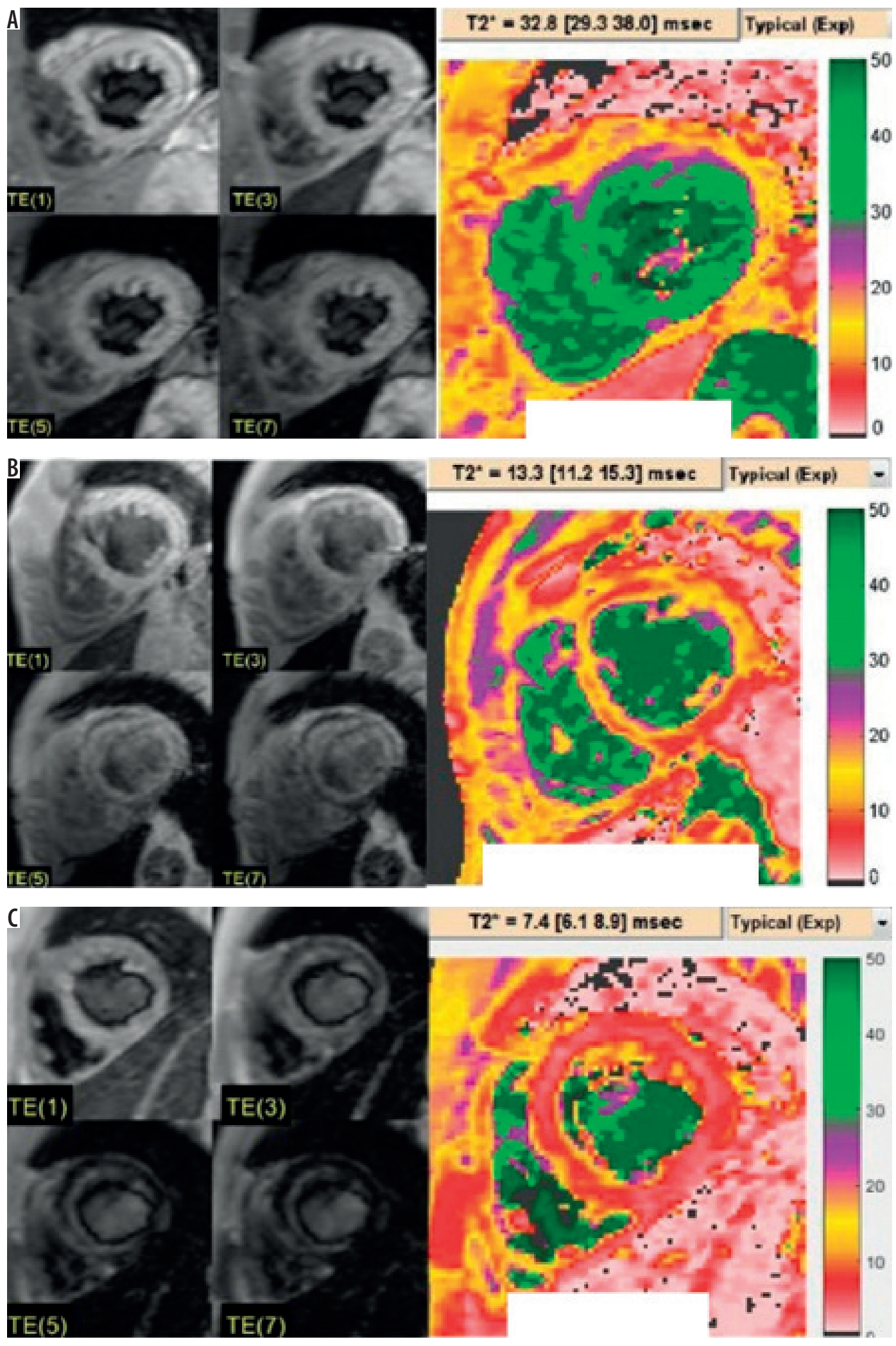

Figure 2. Example of cardiac MRI and T2* colour map from normal (A), mild to moderate (B), and severe (C) iron overload thalassaemia patients

Serum ferritin levels versus myocardial $\mathrm{T}^{*}$ and liver iron concentration values

Serum ferritin ranged between 298 and 14,827. About $17 \%$ of the patients showed mildly elevated ferritin level, while equal percentages (41\%) had moderate and severe high ferritin levels. Myocardial T2* ranged from 48.1 to 7.1 and was at normal level among the majority of the patients (81.4\%), 7.1\% presented with marginal and mild to moderate each, and $4.3 \%$ had severe myocardial iron overload (Table 1). The liver iron concentration ranged from 0.7 to 27.9 , about third of the patients showed mild 
and severe levels (30.0\% and 35.7\%, respectively), moderate liver iron concentration was seen in $21.4 \%$, while only $12.9 \%$ showed normal hepatic iron load (Table 2). Serum ferritin levels showed a significant negative correlation with the liver $\mathrm{T} 2{ }^{\star}$ values $(p=0.01, r=0.318)$. No significant correlation was observed between serum ferritin level and cardiac T2* MRI $(p=0.68, r=0.06)$. Nevertheless, a significant correlation was observed between serum ferritin and liver iron concentration evaluated by MRI $(p=0.04, r=0.68)$.

\section{Discussion}

The assessment of tissue iron is vital for the determination of clinical outcomes, determining when to start chelation, and for selection of the regime that should be prescribed. The quantitative determination of the deposition of iron in various organs in multi-transfused patients with thalassaemia is of extreme importance for the effective monitoring of iron chelation therapy [17]. Until recently, liver iron concentration was quantitated by biopsy, and measurement of ferritin levels patients in steady state has been the only method available to this effect. Nevertheless, ferritin levels alone are not completely acceptable because they may demonstrate considerable variations due to inflammation, infection, or other chronic disorders. Furthermore, there have been instances of heavy iron deposition in which the serum ferritin levels were disproportionately low $[8,9]$. Tissue biopsy is the traditional gold standard for making the diagnosis of myocardial iron overload [18], but iron deposition has a tendency to be patchy $[8,9]$. Endomyocardial biopsies may therefore miss the areas of deposition and provide a false negative result; also, it is an invasive and hazardous procedure [18]. The discomfort and risk that a liver biopsy causes to the patients together with the weak correlation between liver fibrosis and iron concentration reduces the value of this examination, especially when this has to be repeated regularly $[8,11]$. Hence, it is not surprising that alternative, noninvasive, but accurate methods for the assessment of iron deposition have been sought in recent years. Serial assessment of liver and cardiac iron determines that cardiac iron changes lag behind changes in liver iron concentration. The liver is the foremost storage organ for excess iron and willingly acquires excess transferrin- and non-transferrin-bound iron; it also mobilises iron rapidly and proficiently in times of mandate or in response to iron chelation. Conversely, the heart has vigorous mechanisms to prevent excess transfer-mediated uptake [19]. Pathologic myocardial iron overload occurs when the iron binding capacity is saturated and labile free iron species begin to circulate. Even then, cardiac iron uptake is delayed compared to many other extrahepatic organs, including the pancreas. Therefore, many young patients can display severe hepatic iron overloading with no evidence of cardiac iron overloading [20]. $2^{*}$ is an essential MRI relaxation time
Table 1. Relationship between serum ferritin levels and myocardial $T 2^{*}$

\begin{tabular}{|l|c|c|}
\hline Myocardial T2* & Number of patients & Serum ferritin levels \\
\hline Normal & 171 & $1911 \pm 351$ \\
\hline Marginal & 15 & $2084 \pm 443$ \\
\hline Mild to moderate & 15 & $731 \pm 342$ \\
\hline Severe & 9 & $4572 \pm 908$ \\
\hline
\end{tabular}

Table 2. Relationship between serum ferritin levels and liver iron concentration

\begin{tabular}{|l|c|c|}
\hline $\begin{array}{l}\text { Liver iron } \\
\text { concentration }\end{array}$ & Number of patients & Serum ferritin levels \\
\hline Normal & 27 & $1230 \pm 355$ \\
\hline Mild & 63 & $1766 \pm 390$ \\
\hline Moderate & 45 & $2489 \pm 479$ \\
\hline Severe & 75 & $4999 \pm 2033$ \\
\hline
\end{tabular}

that shortens in the presence of iron. It is non-invasive, cost-effective, with no radiation exposure, and has widespread accessibility [21]. Anderson et al. were the first to use the $\mathrm{T} 2{ }^{\star}$ technique for myocardial iron assessment in subjects with thalassaemia major [2]. It was seen that there was a progressive decline in the ejection fraction of the heart as the myocardial iron deposition increased, and all patients with ventricular dysfunction had a myocardial T2* of less than $20 \mathrm{~ms}$ [18]. The purpose of the present study was to reassess the effectiveness of MRI in measuring the amount of iron deposited in the heart and liver of regularly transfused patients with thalassaemia in an endemic area. We included 210 patients with thalassemia major, and all patients were on a regular transfusion program and had been on iron chelation therapy for at least 18 months. The present study also revealed a correlation between liver iron concentration and serum ferritin levels in all patients, which is consistent with the results of Kaltwasser et al. and Fischer [8,22]. No significant correlation was found between serum ferritin levels and myocardial $\mathrm{T} 2{ }^{*}$, and there was a moderate negative correlation between serum ferritin and liver iron concentration evaluated by MRI in our study. Several other studies have found dissimilar correlation strengths, ranging from no correlation to low correlation, between serum ferritin and heart iron content; all have found that serum ferritin cannot satisfactorily evaluate cardiac haemosiderosis $[23,24]$. Such results therefore underscore the importance of MRI T2* as a more accurate method for estimating liver and heart iron overload. Five cases showed severe cardiac overload with mild liver iron overload and no corresponding high serum ferritin levels. There have been reports of heavy cardiac iron deposition in spite of the presence of low serum ferritin levels $[2,25]$. Noetzli et al. disclosed that low values of liver iron or serum ferritin do not certainly signify low-risk of iron-induced cardiomyopathy. This may arise because iron chelation therapy can eliminate iron 
more rapidly from the liver than from the heart, which may normalise liver iron while myocardial iron remains high [7]. T2* has been associated with myocardial and hepatic iron concentration in previous studies, including direct biopsies. Recently, cardiac and liver iron evaluations by MRI have become the primary outcome measures for clinical studies of iron chelation therapy $[21,26]$. Ha et al. [27] stated that liver biopsies are now outdated in thalassaemia cases, except for histological assessment of viral hepatitis. Our study has limitations due to the relatively small number of patients and lack of consideration of prevailing factors that may affect the serum ferritin levels, such as C-reactive protein, white blood cell count, and liver function tests.

\section{Conclusions}

MRI T2* provides a precise, reproducible, and non-invasive technique for measuring tissue iron concentration, sparing the patients from invasive biopsy. Through the widespread application of this technique it should be possible to improve the management of tissue iron overload, and in particular aid the presymptomatic recognition of myocardial siderosis, allowing earlier chelation intensification with its potential for reduced cardiac events.

\section{Acknowledgements}

The authors wish to thank the Faculty of Medicine, Khon Kaen University, and Dr Pairash Saiviroonporn, Department of Radiology, Faculty of Medicine, Mahidol University for their support and substantial contribution to the present study. We would like to acknowledge Yukifumi Nawa for editing the manuscript via Publication Clinic KKU, Thailand.

\section{Conflict of interest}

The authors report no conflict of interest

\section{References}

1. Borgna-Pignatti C, Rugolloto S, De Stefano P, et al. Survival and complications in patients with thalassemia major treated with transfusion and deferoxamine. Haematologica 2004; 89: 1187-1193.

2. Anderson LJ, Holden S, Davis B, et al. Cardiovascular T2-star T2* magnetic resonance for the early diagnosis of myocardial iron overload. Eur Heart J 2001; 22: 2171-2179.

3. Azarkeivan A, Hashmieh M, Akhlaghpoor S, et al. Relation between serum ferritin and liver and heart MRI T2* in beta thalassemia major patients. Eastern Mediterranean Health J 2013; 19: 727-732.

4. Mavrogeni S, Maris T, Gouliamos T, et al. Myocardial iron deposition in B-thalassemia studied by magnetic resonance imaging. Int J Cardiac Imaging 1998; 14: 117-122.

5. Gujja P, Rosing DR, Tripodi DJ, Shizukuda Y. Iron overload cardiomyopathy: better understanding of an increasing disorder. J Am Coll Cardiol 2010; 56: 1001-1012.

6. Eghbali A, Taherahmadi H, Shahbazi M, et al. Association between serum ferritin level, cardiac and hepatic T2-star MRI in patients with major B thalassemia. Iranian J Pediatr Hematol Oncol 2014; 4: 17-21.

7. Fischer R, Longo F, Nielsen P, et al. Monitoring long term efficacy of iron chelation therapy by deferiprone and deferoxamine in patients with beta-thalassemia major application SQUID biomagnetic liver susceptometry. Br J Haematol 2003; 121: 938-948.

8. Ghugre NR, Coates TD, Nelson MD, Wood JC. Mechanisms of tissue-iron relaxivity: nuclear magnetic resonance studies of human liver biopsy specimens. Magn Reson Med 2005; 54: 1185-1193.

9. Roghi A, Cappellini MD, Wood JC, et al. Absence of cardiac siderosis despite hepatic iron overload in Italian patients with thalassemia intermedia: an MRI T2* study. Ann Hematol 2008; 89: 585-589.

10. Wood JC, Tyszka JM, Carson S, et al. Myocardial iron loading in transfusion-dependent thalassemia and sickle cell disease. Blood 2004; 103: 1934-1936.
11. Westwood M, Anderson LJ, Firmin DN, et al. A single breathhold multiecho $\mathrm{T} 2 *$ cardiovascular magnetic resonance technique for diagnosis of myocardial iron overload. J Magn Reson Imaging 2003; 18: 33-39.

12. Liu P, Olivieri N. Iron overload cardiomyopathies: new insights into an old disease. Cardiovasc Drugs Ther 1994; 8: 101-110.

13. Noetzli LJ, Carson SM, Nord AS, et al. Longitudinal analysis of heart and liver iron in thalassemia major. Blood 2008; 112: 2973-2978.

14. Saiviroonporn P, Viprakasit V, Boonyasirinant T, et al. Comparison of the region-based and pixel-wise methods for cardiac $\mathrm{T} 2{ }^{*}$ analysis in 50 transfusiondependent Thai thalassemia patients. J Comput Assist Tomogr 2011; 35: 375-381.

15. Ghugre NR, Enriquez CM, Gonzalez I, et al. MRI detects myocardial iron in the human heart. Magn Reson Med 2006; 56: 681-686.

16. He T, Gatehouse PD, Kirk P, et al. Black-blood T2* technique for myocardial iron measurement in thalassemia. J Magn Reson Imaging 2007; 25: 1205-1209.

17. Jensen PD, Jensen FT, Christensen T, et al. Indirect evidence for the potential ability of magnetic resonance imaging to evaluate the myocardial iron content in patients with transfusional iron overload. MAGMA 2001; 12: 153-166.

18. Voskaridou E, Douskou M, Terpos E, et al. Magnetic resonance imaging in the evaluation of iron overload in patients with beta thalassemia and sickle cell disease. Br J Haematol 2004; 126: 736-742.

19. Walker JM. The heart in thalassemia major. Eur Heart J 2002; 23 : 102-105.

20. Wood JC, Origa R, Agus A, et al. Onset of cardiac iron loading in pediatric patients with thalassemia major. Haematologica 2008; 93 : 917-920.

21. Wood JC, Otto-Duessel M, Aguilar M, et al. Cardiac iron determines cardiac T2*, T2, and T1 in the gerbil model of iron cardiomyopathy. Circulation 2005; 112: 535-543. 
22. Kaltwasser JP, Schalk KP, Hart W. Non-invasive quantitation of liver iron overload by magnetic resonance imaging. Br J Haematol 1990; 741: 360-363.

23. Kirk P, Roughton M, Porter JB, et al. Cardiac T2* magnetic resonance for prediction of cardiac complications in thalassemia major. Circulation 2009; 120: 1961-1968.

24. Selden C, Peters TJ. Separation and assay of iron proteins in needle biopsy specimens of human liver. Clin Chim Acta 1979; 98: 47-52.
25. Oudit GY, Sun H, Triveri MG, et al. L-type Ca2+ channels provide a major pathway for iron entry into cardiomyocytes in iron-overload cardiomyopathy. Nat Med 2003; 9: 1187-1194.

26. Wood JC, Kang BP, Thompson A, et al. The effect of deferasirox on cardiac iron in thalassemia major: impact of total body iron stores. Blood 2010; 116: 537-543.

27. Ha SY, Chik KW, Ling SC, et al. A randomized controlled study evaluating the safety and efficacy of deferiprone treatment in thalassemia major patients from Hong Kong. Hemoglobin 2006; 30: 263-274. 